\title{
Prevalência e intensidade da sede de crianças no pós-operatório imediato
}

\author{
Prevalence and thirst intensity in children in the immediate postoperative period
}

Prevalencia e intensidad de la sed de niños en el posoperatorio inmediato

\author{
Andressa Riviera ${ }^{1}$ iD https://orcid.orgy0000-0002-6775-3061 \\ Isadora Pierotti1 î https://orcid.org//0000-0001-8050-8389 \\ Carla Regina Lodi de Mello ${ }^{1}$ ib htps://orcid.org/0000-0002-6417-0549 \\ Marcela Maria Birolim ${ }^{1}$ if https://orcid.org/0000-0001-6976-4955 \\ Lígia Fahl Fonseca ${ }^{1}$ io https:/orcid.org/0000-0001-7550-9141
}

Como citar:

illo CR, Birolim MM, Fonseca

LF. Prevalência e intensidade da sede de crianças

no pós-operatório imediato. Acta Paul Enferm. 2022;35:eAPE02931.

DOI

http://dx.doi.org/10.37689/acta-ape/2022A002931

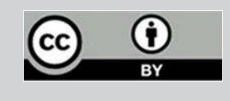

Descritores

Sede; Criança; Enfermagem perioperatória; Cuidados pósoperatórios; Enfermagem pediátrica

Keywords

Thirst; Child; Perioperative nursing; Postoperative care;

Pediatric nursing

Descriptores

Sed; Niño; Enfermería perioperatoria; Cuidados posoperatorios; Enfermería pediátrica

Submetido 18 de Outubro de 2019

Aceito

21 de Maio de 2021

Autor correspondente

Carla Regina Lodi de Mello E-mail: carla-lodi@hotmail.com

Editor Associado (Avaliação pelos pares): Denise Myuki Kusahara

(https://orcid.org/0000-0002-9498-0868) Escola Paulista de Enfermagem, Universidade Federal de São Paulo, São Paulo, SP, Brasil

\section{Resumo}

Objetivo: Identificar a prevalência e intensidade da sede de crianças no pós-operatório imediato e seus fatores associados.

Métodos: Estudo transversal e analítico. A amostra consistiu-se de 78 crianças de quatro a doze anos em sala de recuperação anestésica no pós-operatório imediato. A presença de sede, bem como seus atributos e sinais foi identificada por questionamento da pesquisadora e/ou autorrelato da criança e seu cuidador. A intensidade de sede foi mensurada por meio da escala de faces. As variáveis desfecho foram presença e intensidade da sede. A razão de prevalência foi calculada por regressão de Poisson, com variância robusta.

Resultados: A prevalência de sede foi de $88,5 \%$ sendo que 39,7\% referiram sede no pós-operatório e $48,7 \%$ desde 0 pré-operatório. Quanto à intensidade, 20,5\% referiram sede forte e 37,2 \% sede intensa. Adicionalmente, mais da metade das crianças (59\%) a verbalizou de forma espontânea. Os fatores associados à maior intensidade da sede foram: sexo feminino $(R P=1,27)$; queixa espontânea $(R P=1,29)$; referir sensação de boca seca $(\mathrm{RP}=1,93)$ e de saliva grossa $(\mathrm{RP}=1,43)$; a idade apresentou associação inversa com a intensidade da sede, ou seja, quanto menor a idade, maior a intensidade da sede (beta= $-0,053 ; p=0,01$ ).

Conclusão: A sede na criança cirúrgica apresenta elevada prevalência e intensidade. A criança é capaz de identificar os sinais relacionados à sede e a verbaliza espontaneamente. Sexo, queixa espontânea, idade, boca seca e saliva grossa apresentaram associação com a intensidade. Estes resultados sinalizam a necessidade de intervenções intencionais para reduzir a sede da criança na prática clínica.

\section{Abstract}

Objective: To identify the prevalence and thirst intensity in children in the immediate postoperative period and its associated factors.

Methods: This is a cross-sectional and analytical study. The sample consisted of 78 children aged four to twelve years in the post-anesthesia care unit in the immediate postoperative period. Thirst presence, as well as its attributes and signs, were identified by questioning by the researcher and/or self-report of children and their caregivers. Thirst intensity was measured using the face scale. The outcome variables were thirst presence and intensity. Prevalence ratio was calculated by Poisson regression, with robust variance.

Results: The prevalence of thirst was $88.5 \%$, with $39.7 \%$ reporting thirst in the postoperative period and $48.7 \%$ since the preoperative period. As for the intensity, $20.5 \%$ reported strong thirst and $37.2 \%$ intense thirst. Additionally, more than half of the children (59\%) reported it spontaneously. The factors associated with greater thirst intensity were: female sex $(P R=1.27)$; spontaneous complaint $(P R=1.29)$; reporting feeling of dry mouth $(P R=1.93)$ and thick saliva $(P R=1.43)$; age was inversely associated with thirst intensity, i.e., the younger the age, the greater the thirst intensity (beta $=-0.053 ; p=0.01$ ). 
Conclusion: Thirst in surgical children has a high prevalence and intensity. Children are able to identify the signs related to thirst and spontaneously reports it. Sex, spontaneous complaints, age, dry mouth and thick saliva were associated with intensity. These results signal the need for intentional interventions to reduce child thirst in clinical practice.

\section{Resumen}

Objetivo: Identificar la prevalencia e intensidad de la sed de niños en el posoperatorio inmediato y los factores asociados.

Métodos: Estudio transversal y analítico. La muestra fue formada por 78 niños de 4 a 12 años en sala de recuperación anestésica en el posoperatorio inmediato. La presencia de sed, así como sus atributos y señales, fue identificada mediante cuestionario de la investigadora 0 autorrelato del niño y su cuidador. La intensidad de la sed fue medida mediante escala de expresiones faciales. Las variables de resultado fueron presencia e intensidad de la sed. La razón de prevalencia fue calculada por regresión de Poisson, con varianza robusta.

Resultados: La prevalencia de sed fue del 88,5 \% de los cuales el 39,7 \% relató sed en el posoperatorio y el 48,7 \% desde el preoperatorio. Con relación a la intensidad, el 20,5 \% relató sed fuerte y el 37,2 \% sed intensa. Además, más de la mitad de los niños (59 \%) la verbalizó de forma espontánea. Los factores asociados con una mayor intensidad de sed fueron: sexo femenino ( $R P=1,27)$, queja espontánea $(R P=1,29)$, relatar sensación de boca seca ( $R P=1,93)$ y de saliva espesa $(\mathrm{RP}=1,43)$, la edad presentó asociación inversa respecto a la intensidad de la sed, es decir, cuanto menor la edad, mayor la intensidad de la sed (beta $=-0,053 ; p=0,01)$.

Conclusión: La sed en niños quirúrgicos presenta una elevada prevalencia e intensidad. Los niños son capaces de identificar las señales relacionadas con la sed y la verbalizan espontáneamente. Sexo, queja espontánea, edad, boca seca y saliva espesa presentan asociación con la intensidad. Estos resultados indican la necesidad de intervenciones intencionales para reducir la sed de los niños en la práctica clínica.

\section{Introdução}

Os procedimentos cirúrgicos podem resultar em inúmeros desconfortos à criança em todo o perioperatório. Este período é composto por pré-operatório, transoperatório e pós-operatório. O pré-operatório engloba desde a notícia da realização da cirurgia até 24 horas que antecedem o ato cirúrgico. $\mathrm{O}$ transoperatório refere-se ao momento que o paciente é recebido no centro cirúrgico até a saída da sala de operação. O período pós-operatório é classificado em imediato, mediato e tardio. Imediato, compreende as primeiras 24 horas após o término do procedimento cirúrgico, incluindo o tempo de permanência em sala de recuperação anestésica. $\mathrm{O}$ período mediato inicia-se após as primeiras 24 horas iniciais ao procedimento. $\mathrm{O}$ pós-operatório tardio, ocorre após 15 dias até um ano da realização do procedimento anestésico-cirúrgico. ${ }^{(1)}$

No pré-operatório, alguns preparos inerentes ao ato anestésico cirúrgico devem ser realizados. Destaca-se o jejum, indicado com a finalidade de proteger a criança de riscos de aspiração de conteúdo gástrico regurgitado por perda de reflexos de proteção das vias aéreas durante o procedimento anestésico. ${ }^{(2)}$ Embora o estômago da criança deva estar livre de sólidos antes da cirurgia, é importante não interromper a ingestão hídrica por mais tempo que o necessário. As recomendaçôes são de duas horas para líquidos claros, quatro para leite materno, seis para fórmula láctea e refeições leves e oito para refeição gordurosa. ${ }^{(3)}$ Todavia, na prática clínica, esse tempo é excessivamente prolongado, chegando a 11 horas, sendo, portanto, prejudicial para as crianças. $^{(4)}$

Dentre os prejuízos que o jejum prolongado pode acarretar ressalta-se a sede. Trata-se de um desconforto prevalente neste período para a criança, com taxas de 58\% ${ }^{(5)}$ e 66\%. ${ }^{(6)}$ Embora apresente prevalência elevada e gere desconforto para a criança, ${ }^{(7)}$ a sede ainda é um tema pouco explorado e valorizado na assistência à criança cirúrgica.

O mecanismo fisiológico que desencadeia a sede e saciedade na criança assemelha-se ao do adulto. Entre as diferenças há que se destacar: maior área de superfície em relação à massa corporal; menor tolerância ao calor, dado que a criança transpira mais, desidratando-se mais facilmente. ${ }^{(8)}$ Essas diferenças acarretam nas crianças maior necessidade de líquidos em relação aos adultos.

$\mathrm{Na}$ criança cirúrgica, a sede é deflagrada por diversos fatores: jejum prolongado, ansiedade, temor do desconhecido, além do estresse inerente ao procedimento. ${ }^{(6,9,10)} \mathrm{O}$ estresse cirúrgico, náusea e hipoglicemia estimulam a secreção do hormônio antidiurético $(\mathrm{ADH})$, portanto também desencadeiam sensação de sede. ${ }^{(11-13)}$ No intraoperatório, a intubação e o uso de medicamentos como opioides e anticolinérgicos, acarretam desidratação da mucosa oral, gerando sensação de boca seca, culminan- 
do com liberação de ADH. ${ }^{(11-13)}$ A dificuldade da criança em se expressar de maneira adequada, muitas vezes constitui-se em um desafio para a identificação da sede. No entanto, podemos detectá-la pelo autorrelato, assim como, por meio de sinais físicos como a boca seca, lábios esbranquiçados e movimento de umidificação dos lábios com a língua. ${ }^{(7)}$

O período pós-operatório imediato (POI), também é caracterizado pela presença de fatores estressantes para a criança, como a sede, constituindo-se um dos principais fatores responsáveis pela angústia vivenciada neste período. ${ }^{(4)}$ Embora se observe com clareza a presença e desconforto da sede na vivência prática do cuidado à criança em recuperação anestésica, não se localizaram estudos que abordassem esse tema com intencionalidade, bem como, sua prevalência neste período.

Outras complicações como dor, hipotermia, náusea e vômito são extensivamente pesquisadas. A sede, no entanto, não faz parte de guidelines e protocolos de cuidados à criança no POI. Alguns estudos analisam a sede somente sob a perspectiva do jejum prolongado ${ }^{(5,6,9,10)}$ não investigando outros fatores relacionados à ela, assim como a percepção da criança sobre esse sintoma em Sala de Recuperação Anestésica (SRA). Adicionalmente, esses estudos são focados no período pré-operatório. Há, portanto, escassez de evidências sobre a sede da criança no POI.

Reconhecer a presença e relevância deste sintoma, portanto, pode contribuir para a valorização da identificação e tratamento da sede em SRA. Constata-se, assim, a necessidade da realizaçáo da presente pesquisa, com o objetivo de identificar a prevalência e intensidade da sede de crianças no POI e seus fatores associados.

\section{Métodos}

Trata-se de um estudo transversal e analítico, realizado em hospital-escola púbico de grande porte no norte do Paraná, com 313 leitos para internaçáo do Sistema Único de Saúde. A coleta de dados aconteceu na SRA do centro cirúrgico (CC) da instituição em questão. O CC possui sete salas, onde são realizadas, em média, 150 cirurgias infantis por trimestre.
Possui uma SRA geral cujo espaço físico de recuperação é o mesmo que dos adultos. Conta com equipamentos e recursos específicos para atendimento da criança e profissionais especializados que periodicamente participam de treinamentos referentes à assistência pós-operatória. Inclui-se a mãe ou responsável no processo de recuperação, assim que a criança recupera a consciência. A coleta de dados ocorreu entre $1^{\circ}$ de fevereiro e 31 de maio de 2016. A população desse estudo foi composta por crianças de quatro a doze anos, de ambos os sexos, submetidas a procedimento anestésico-cirúrgico sob quaisquer técnicas anestésicas, clínicas cirúrgicas e tempo de duração. Os critérios de inclusão foram: estar no POI, na SRA, no período das sete às dezenove horas em dias úteis; estar orientada e consciente, com índice seis na escala de Steward. ${ }^{(14)}$ Determinou-se a faixa etária a partir de quatro anos, quando a criança já identifica sequências envolvendo mais de um objeto, demonstrando compreensão elementar de verbos, identificando diferenças em figuras e relatando histórias conhecidas sem figuras. ${ }^{(15)}$ Os critérios de exclusão foram: estado de agitação e choro intenso (características sugestivas de delirium) e, crianças especiais (com alteração do estado neurológico e sem possibilidade efetiva de comunicação). $\mathrm{O}$ tamanho da amostra foi não probabilística, determinada por tempo de coleta. Os dados clínicos foram obtidos do prontuário; as informaçóes sobre tempo de jejum pré-operatório e início da sede foram relatadas pela criança, pais ou responsáveis no pré ou pós-operatório. A abordagem da criança foi realizada no POI, na SRA, quando a criança alcançava o índice seis de Steward. Esse índice avalia três domínios: vias aéreas pérvias; consciência; movimentação. A pontuação varia de 0 a 6 sendo que cada um dos três domínios pode ser pontuado de 0 a 2 , desta forma, o menor valor é indicativo de que o paciente ainda não está recuperado, e o maior valor, já recuperado da anestesia. ${ }^{(14)}$ Após a interação, a pesquisadora questionava a criança sobre presença de sede, quando ela já não o tivesse se manifestado sobre a mesma, espontaneamente. Em caso positivo, solicitava-se à criança que quantificasse sua sede, apontando para a escala visual de faces adaptada, ${ }^{(16)}$ sendo que zero indicava sem sede, e quatro, sede 
insuportável. Optou-se por utilizar a escala de faces, considerando que se trata de uma escala visual analógica, sendo estas, extensivamente utilizadas em investigaçóes que mensuram a sede. ${ }^{(17)} \mathrm{O}$ diferencial da escala de faces é o uso de personagens brasileiros Mônica e Cebolinha, que apresentam familiaridade por parte das crianças. Além disso, a escala de faces é utilizada para avaliar a presença de outro sintoma subjetivo na criança, a dor. ${ }^{(16)}$ Uma vez que a sede também é um sintoma subjetivo e autorrelatado, optou-se pela utilização desta escala analógica para mensuração de sua intensidade. Adicionalmente, questionava-se a criança sobre a presença de sinais percebidos por ela referentes à sede: boca seca, garganta seca, lábio rachado, língua grossa e saliva grossa. As variáveis de desfecho foram presença e intensidade da sede; as preditoras foram: sexo, idade, jejum pré-operatório, classificação do estado físico de acordo com a American Society of Anesthesiologists (ASA), uso de opióides, intubação endotraqueal, tipo de técnica anestésica, procedimento cirúrgico, tempo de anestesia, queixa espontânea de sede, início da sede, sinais e sintomas da sede. ${ }^{(12,13)}$ Para a tabulação e análise dos dados foi utilizado o software Statistical Package for the Social Sciences (SPSS), versão 20.0, com a realização de dupla digitação, para maior segurança. Foram realizadas análises descritivas com a apresentação das frequências absolutas e relativas para as variáveis categóricas, e médias, mediana, desvio padrão, valores mínimos e máximos para as variáveis contínuas. Fez-se análise bivariada para verificar associação entre presença e intensidade da sede e variáveis preditoras. Todas as variáveis com $\mathrm{p}<0,20$, na análise bivariada, foram selecionadas para compor o modelo de regressão multivariado pelo método backward. Calculou-se a razão de prevalência (RP) por regressão de Poisson, com variância robusta e respectivos intervalos de confiança (IC95\%). Permaneceram no modelo final as variáveis preditoras que mantiveram associação significativa, após o ajuste $(\mathrm{p}<0,05)$, de acordo com o teste de Wald. Obteve-se a assinatura do Termo de Consentimento do responsável e Termo de Assentimento da criança no pré-operatório. $\mathrm{O}$ Comitê de Ética em Pesquisa da Instituição aprovou o projeto de pesquisa, sob o protocolo n ${ }^{\circ}$. CAAE:
29069414.5.0000.5231, de 15/04/2014, conforme determina a Resolução 466/2012, do Conselho Nacional de Saúde. O presente estudo foi o resultado de uma dissertaçáo de mestrado.

\section{Resultados}

No período de estudo, realizaram-se 231 cirurgias infantis de diversas especialidades, das quais 21 crianças fizeram a recuperação na unidade de terapia intensiva, 5 não faziam contato por comprometimento cognitivo, 122 passaram por cirurgias fora do horário e dia estabelecidos e 5 consumiram água ou gelatina antes de se aplicar o instrumento. A amostra, portanto, foi constituída de 78 crianças, sendo a maioria do sexo masculino $(70,5 \%)$, com idade entre 4 e 12 anos (média =7,6; dp 2,7); as clínicas prevalentes foram: cirurgia infantil (59\%), ortopedia $(19,2 \%)$, otorrinolaringologia $(10,3 \%)$ e outras especialidades (11,5\%). Do total, $82,1 \%$ dos casos foi classificado como ASA I e 17,9\% como ASA II; o tempo de jejum pré-operatório variou de 1,5 a 72 horas, com média de 13,48 horas $(d p 8,6)$ (Tabela 1).

A presença de sede ocorreu em 88,5\% (69) dos casos, a verbalização foi espontânea para 59\% (46) das crianças, sendo que 39,7\% (31) referiram sede no pós-operatório e 48,7\% (38) desde o pré-operatório. Em relação à intensidade, $11,5 \%$ verbalizaram sede leve, $19,5 \%$ moderada, $20,5 \%$ forte e $37,0 \%$ intensa. Das 69 crianças que referiram sede, $74,4 \%$ queixaram-se de boca seca, $19,2 \%$ de garganta seca, $20,5 \%$ de saliva grossa e $47,8 \%$ apresentaram combinação de dois ou mais atributos da sede (Tabela 2).

Inicialmente se fez análise bivariada entre as variáveis de desfecho "presença e intensidade da sede". Para o desfecho presença da sede, não foi encontrada associação significativa com as variáveis preditoras: sexo, idade, jejum, índice de ASA, uso de opióides, intubação, tipo de técnica anestésica, procedimento cirúrgico, queixa espontânea de sede e atributos da sede.

$\mathrm{Na}$ análise bivariada realizada entre a intensidade da sede e as variáveis clínico-cirúrgicas, entra- 
Tabela 1. Distribuição da amostra em relação às variáveis clínico-cirúrgicas de crianças em sala de recuperação anestésica $(\mathrm{n}=78)$

\begin{tabular}{|c|c|c|c|c|c|}
\hline Variáveis & Média & $\begin{array}{l}\text { Desvio- } \\
\text { Padrão }\end{array}$ & Mínimo & Máximo & IC $95 \%$ \\
\hline Idade (em anos) & 7,65 & 2,70 & 4 & 12 & $7,11-8,20$ \\
\hline Tempo de jejum (em horas) & 13,48 & 8,60 & 1,5 & 72 & $11,75-15,21$ \\
\hline $\begin{array}{l}\text { Tempo de anestesia (em } \\
\text { minutos) }\end{array}$ & 94,58 & 6,36 & 10 & 370 & $81,90-107,25$ \\
\hline \multirow{2}{*}{$\begin{array}{l}\text { Tempo do procedimento } \\
\text { (em minutos) }\end{array}$} & 70,32 & 5,49 & 5 & 240 & $59,39-81,25$ \\
\hline & & & $\mathrm{n}(\%)$ & & IC 95\% \\
\hline \multicolumn{6}{|l|}{ Sexo } \\
\hline Masculino & & & $55(70,50)$ & & $58,95-80,02$ \\
\hline Feminino & & & $23(29,50)$ & & $19,97-41,04$ \\
\hline \multicolumn{6}{|l|}{ Especialidades } \\
\hline Cirurgia Infantil & & & $46(59,00)$ & & $47,25-69,80$ \\
\hline Ortopedia & & & $15(19,20)$ & & $11,50-30,04$ \\
\hline Otorrinolaringologia & & & $8(10,30)$ & & $04,84-19,72$ \\
\hline Outros & & & $9(11,50)$ & & $05,73-21,25$ \\
\hline \multicolumn{6}{|l|}{ ASA } \\
\hline । & & & $64(82,10)$ & & $71,37-89,49$ \\
\hline$\|$ & & & $14(17,90)$ & & $10,50-28,62$ \\
\hline \multicolumn{6}{|l|}{ Intubação } \\
\hline Sim & & & $64(82,10)$ & & $71,37-89,49$ \\
\hline Não & & & $14(17,90)$ & & $10,50-28,62$ \\
\hline \multicolumn{6}{|l|}{ Sulfato de atropina } \\
\hline Sim & & & $33(42,30)$ & & $31,36-54,00$ \\
\hline Não & & & $45(57,70)$ & & $45,99-68,63$ \\
\hline \multicolumn{6}{|l|}{ Citrato de fentanila } \\
\hline Sim & & & $40(51,30)$ & & $37,77-62,65$ \\
\hline Não & & & $38(48,70)$ & & $7,34-60,22$ \\
\hline \multicolumn{6}{|l|}{ Cloridrato de cetamina } \\
\hline Sim & & & $3(3,80)$ & & $00,99-11,59$ \\
\hline Não & & & $75(96,20)$ & & $88,40-99,00$ \\
\hline \multicolumn{6}{|l|}{ Cloridrato de midazolan } \\
\hline Sim & & & $9(11,50)$ & & $05,73-21,25$ \\
\hline Não & & & $69(88,50)$ & & $78,74-94,26$ \\
\hline \multicolumn{6}{|l|}{ Sulfato de morfina } \\
\hline Sim & & & $18(23,10)$ & & $14,59-34,24$ \\
\hline Não & & & $60(76,90)$ & & $65,75-85,40$ \\
\hline
\end{tabular}

ram no modelo multivariado as seguintes variáveis $(\mathrm{p}<0,20)$ : sexo $(\mathrm{p}=0,012)$, utilização da atropina $(\mathrm{p}=$ $0,167)$, queixa espontânea da sede $(p=0,000)$, percepção de boca seca $(\mathrm{p}=0,004)$, percepção de saliva grossa $(p=0,005)$ e idade $(p=0,011)$. Permaneceram no modelo final as variáveis descritas na Tabela 3.

Os fatores associados à maior intensidade da sede foram: sexo feminino ( $R P=1,27$ IC 95\%: 1,05-1,54); queixa espontânea da sede $(\mathrm{RP}=1,29$ IC95\%:1,04-1,60); referir sensação de boca seca $(\mathrm{RP}=1,92$ IC95\%: 1,33-2,80) e de saliva grossa ( $\mathrm{RP}=1,43$ IC 95\%: 1,15-1,78); a idade apresentou associação inversa com a intensidade da sede, ou seja, quanto menor a idade, maior a intensidade da sede (beta $=-0,053 ; \mathrm{p}=0,01)$ (Tabela 3$)$.

Tabela 2. Distribuição da amostra em relação às variáveis queixa espontânea, presença, início, intensidade e atributos da sede de crianças em sala de recuperação anestésica $(n=78)$

\begin{tabular}{|c|c|c|}
\hline Variáveis & $n(\%)$ & IC $95 \%$ \\
\hline \multicolumn{3}{|c|}{ Queixa espontânea de sede } \\
\hline Sim & $46(59,00)$ & $47,25-69,80$ \\
\hline Não & $32(41,00)$ & $30,19-52,74$ \\
\hline \multicolumn{3}{|c|}{ Presença de sede } \\
\hline Sim & $69(88,50)$ & $78,74-94,26$ \\
\hline Não & $9(11,50)$ & $05,73-21,25$ \\
\hline \multicolumn{3}{|l|}{ Início da sede } \\
\hline Pré-cirúrgico & $38(48,70)$ & $37,34-60,22$ \\
\hline Pós-cirúrgico & $31(39,70)$ & $29,02-51,47$ \\
\hline Sem sede & $9(11,60)$ & $05,73-21,25$ \\
\hline \multicolumn{3}{|c|}{ Intensidade da sede } \\
\hline 0 Nenhum & $9(11,50)$ & $05,73-21,25$ \\
\hline 1 Leve & $9(11,50)$ & $05,73-21,25$ \\
\hline 2 Moderada & $15(19,50)$ & $11,50-30,04$ \\
\hline 3 Forte & $16(20,50)$ & $12,52-31,45$ \\
\hline 4 Intensa & $29(37,00)$ & $26,71-48,91$ \\
\hline \multicolumn{3}{|l|}{ Boca seca } \\
\hline Sim & $58(74,40)$ & $63,00-83,28$ \\
\hline Não & $20(25,60)$ & $16,71-36,99$ \\
\hline \multicolumn{3}{|l|}{ Garganta seca } \\
\hline Sim & $15(19,20)$ & $11,50-30,04$ \\
\hline Não & $63(80,80)$ & $69,95-88,49$ \\
\hline \multicolumn{3}{|l|}{ Lábio rachado } \\
\hline Sim & $6(7,70)$ & $03,16-16,58$ \\
\hline Não & $72(92,30)$ & $83,41-96,83$ \\
\hline \multicolumn{3}{|l|}{ Língua grossa } \\
\hline Sim & $11(14,10)$ & $07,58-24,25$ \\
\hline Não & $67(85,90)$ & $75,74-92,41$ \\
\hline \multicolumn{3}{|l|}{ Saliva grossa } \\
\hline Sim & $16(20,50)$ & $12,52-31,45$ \\
\hline Não & $62(79,50)$ & $68,54-87,47$ \\
\hline
\end{tabular}

Tabela 3. Fatores associados à maior intensidade da sede de crianças em sala de recuperação anestésica $(n=78)$

\begin{tabular}{|c|c|c|c|c|}
\hline \multirow{3}{*}{ Variáveis } & \multirow{3}{*}{$\begin{array}{l}\text { Razão de } \\
\text { prevalência }\end{array}$} & \multirow{2}{*}{\multicolumn{2}{|c|}{$\begin{array}{c}\text { Intensidade da sede } \\
\text { Intervalo de } \\
\text { confiança 95\% }\end{array}$}} & \multirow{3}{*}{$p$-value } \\
\hline & & & & \\
\hline & & Inferior & Superior & \\
\hline \multicolumn{5}{|l|}{ Sexo } \\
\hline Feminino & 1,27 & 0,04 & 5,71 & 0,017 \\
\hline Masculino & 1,00 & . & . & . \\
\hline \multicolumn{5}{|c|}{ Queixa espontânea de sede } \\
\hline Sim & 1,29 & 0,03 & 0,47 & 0,022 \\
\hline Não & 1,00 & . & . & . \\
\hline \multicolumn{5}{|l|}{ Boca seca } \\
\hline Sim & 1,92 & 0,28 & 1,03 & 0,001 \\
\hline Não & 1,00 & . & . & . \\
\hline \multicolumn{5}{|l|}{ Saliva grossa } \\
\hline Sim & 1,43 & 0,14 & 0,57 & 0,001 \\
\hline Não & 1,00 & . & . & . \\
\hline Idade (em anos) & 0,95 & $-0,095$ & $-0,01$ & 0,015 \\
\hline
\end{tabular}




\section{Discussão}

Verificou-se que a temática da sede no POI de crianças constitui-se em abordagem pouco explorada na literatura científica. $\mathrm{O}$ presente estudo inova com a investigação deste tema em crianças com idade superior a quatro anos em SRA. A análise de fatores relacionados a presença e intensidade da sede, traz compreensão adicional a respeito da prevalência desse sintoma em crianças cirúrgicas. Ademais, foram exploradas as percepçóes das crianças quanto aos atributos inerentes à sede, como boca seca, saliva grossa, garganta seca, lábio rachado, língua grossa.

A prevalência da sede neste estudo foi elevada sendo que mais da metade das crianças a expressaram espontaneamente. A referência à sede foi, muitas vezes, a primeira verbalização ao despertar da anestesia, com demonstrações de choro, agitação, desconforto e repetidas solicitaçóes por água. A sede torna-se perceptível à medida que as crianças recobram propriocepção e habilidade de identificar estímulo ambiental durante a recuperação da anestesia. $\mathrm{O}$ paciente adulto também cita a sede como primeiro desconforto intenso logo ao acordar da anestesia. ${ }^{(18)}$ Para a criança, essa sensação pode ser intensificada pela desorientação gerada pelos anestésicos e ansiedade em razão do ambiente desconhecido. Além das alteraçóes de níveis de consciência inerentes ao período de recuperação anestésica, a privação de água pode desencadear variaçóes do cognitivo, acarretar irritabilidade na criança. ${ }^{(15,19,20)}$

Estudo revelou prevalência de sede no pré-operatório superior à encontrada nesta pesquisa, chegando a $66 \%$. ${ }^{(3)}$ Em outra investigação realizada com cuidadores de crianças com idade entre 0 e 3 anos, foi identificada presença de sede em 58,9\%, e as crianças com idade superior a 3 anos autorelataram sua sede com prevalência de $58 \%{ }^{(5)}$ Esses resultados indicam que a sede é uma experiência vivenciada por muitas crianças que se submetem a uma cirurgia.

Houve associação positiva entre a intensidade da sede e sua verbalização. A intensidade da sede foi maior em crianças que se queixaram espontaneamente em relação àquelas que não se queixaram. Estudos realizados com adultos revelaram re- lato espontâneo de sede entre $12 \%{ }^{(21)}$ e $38,3 \% .^{(18)}$ Observa-se, portanto que a criança apresenta menos filtros expressando seu desconforto de forma espontânea com mais frequência.

Ao mesmo tempo, percebe-se que muitas crianças que sentem sede não a verbalizam, a menos que sejam questionadas. Discute-se que as orientaçóes dadas à criança quanto à impossibilidade de ingerir água de qualquer forma desde o pré-operatório interfiram em sua coragem de verbalizar sede. ${ }^{(7)}$ A percepção de um sintoma desagradável é influenciada por cultura, conhecimento e orientaçóes recebidas. Experiências anteriores também podem afetar sua reação e expressão quando experienciam a sede. ${ }^{(22,23)}$

Notou-se nos resultados que mais da metade das crianças relataram sede forte ou intensa, indicando a magnitude desse desconforto. Outros estudos avaliaram a intensidade de sede na criança cirúrgica no pré-operatório. ${ }^{(5,6)} \mathrm{Um}$ deles mostrou que $27 \%$ das crianças referiram sentir muita sede, sendo o tempo médio de jejum para líquido aproximadamente de 8 horas. ${ }^{(6)}$ Em outra pesquisa, $23,8 \%$ das crianças com idade superior a três anos, relataram sede intensa, e o tempo médio de jejum para líquido foi de 9,4 horas. ${ }^{(5)}$

A idade apresentou associação inversa com a intensidade da sede, ou seja, quanto menor a idade, maior a intensidade da sede. $\mathrm{O}$ teor de água corporal diminui com o aumento da idade. Assim as crianças menores possuem menor tolerância à falta de água. Em caso de desidratação, mesmo leve, a criança pode apresentar cansaço, dores de cabeça, diminuição da concentração, irritabilidade e pele seca. ${ }^{(8,24)}$

A identificação do início da sede no pré-operatório ocorreu em quase metade das crianças, efetuada pelos pais ou cuidadores. No perioperatório, pais e cuidadores vivenciam com a criança o período de jejum $^{(5,7)}$ e conseguem identificar e relatar sinais e sintomas da sede entre os quais: cavidade oral e lábios ressecados e esbranquiçados, constante deglutição de saliva, dor na garganta e comportamento de busca por água quando os lábios são umedecidos por alguém. ${ }^{(7)}$

$\mathrm{O}$ resultado do presente estudo evidenciou que o tempo de jejum a que a criança é submetida é 
muito maior do que o recomendado, corroborando dados encontrados em outras realidades. ${ }^{(2)}$ Pesquisa realizada na França encontrou tempo de jejum pré-operatório de aproximadamente 11 horas. ${ }^{(4)}$ Outro estudo, realizado no Canadá, revelou que o tempo de jejum pré-operatório foi maior do que o recomendado para $70 \%$ das crianças, e para $34 \%$ delas, foi recomendado a partir da meia noite. ${ }^{(25)}$ Em hospital na Suíça, o tempo de jejum para líquidos claros variou de 1,1 a 15,5 horas. ${ }^{(26)}$

A manutenção do jejum prolongado com o intuito de proteger a criança é paradoxal frente às evidências de vários estudos que advogam a abreviação do tempo de restrição hídrica. ${ }^{(10,20)} \mathrm{A}$ ingestão de líquidos claros duas horas antes do procedimento cirúrgico não resulta em maiores volumes gástricos ou valores de $\mathrm{pH}$ gástrico menores em crianças. (10) Adicionalmente, quando incentivadas a ingerir líquidos claros até duas horas antes da cirurgia, a criança apresenta menor intensidade de sede durante 24 horas do pós-operatório. ${ }^{(27)}$ Recentes evidências indicam que é seguro para a criança ingerir líquidos claros até mesmo somente uma hora antes de anestesia geral. ${ }^{(20)}$ Esses resultados nos alertam para a necessidade de mudar a prática de jejum prolongado que é realizada em muitas instituições sem evidências científicas, fundamentada em hábitos e rotinas institucionais.

Reconhece-se a mera comunicação do estado de jejum como um estímulo não regulatório para o início da sede. ${ }^{(23)}$ Neste estudo praticamente a totalidade das crianças foram mantidas em jejum por períodos superiores a oito horas. Manter uma criança por 72 horas em jejum no pré-operatório não é compatível com os princípios do cuidado humanizado, e essa prática precisa ser revista nas instituiçóes de saúde.

A sede é um sintoma multifatorial e se expressa por diversos atributos. A maneira como cada criança a percebe é individual e sofre influência de seu estado de saúde e de características pessoais e ambientais. ${ }^{(22,23,28)}$ No presente estudo, a criança, quando questionada, foi competente para identificar e verbalizar a percepção de seus atributos: boca seca, lábio rachado, língua grossa, saliva grossa. Estudo realizado com pacientes adultos em SRA identificou a prevalência de atributos semelhantes que compuseram uma escala de avaliação de desconforto da sede perioperatória. ${ }^{(29)}$ Mesmo na ausência de verbalização por parte da criança, é importante que se avaliem intencionalmente os atributos que podem indicar a presença da sede.

A razão de prevalência de intensidade da sede foi maior em crianças que referiram boca seca do que naquelas que não referiram. A intensidade da sede foi maior em crianças que referiram saliva grossa em relação àquelas que não referiram. A sede também envolve uma dimensão motivacional e afetiva que inclui o desejo por água e aversão à sensação de boca seca - talvez o atributo mais perceptivo para adultos e crianças. ${ }^{(28)}$ Pacientes cirúrgicos adultos mencionam a sensação de boca seca como algo extremamente aversivo, comparando-a a ingestão de um tubo de cola. ${ }^{(30)}$

Neste estudo, encontrou-se razão de prevalência de intensidade de sede em meninas maior que em meninos. Crianças diferem dos adultos no conteúdo de água corporal total, e meninos e meninas diferem no conteúdo de água do corpo com a maturação. Geralmente meninos de todas as idades tendem a ter maiores proporçóes de água corporal total que meninas. ${ }^{(31)}$

Este estudo abordou a identificação e intensidade do sintoma sede pelas crianças no POI, além de identificar os atributos por elas percebidos. Precisamos ainda avançar na compreensão das repercussóes físicas e emocionais da sede para a criança cirúrgica, assim como na adoção de estratégias para seu manejo.

A limitação deste estudo foi a utilização de amostra não probabilística, reduzindo sua validade externa. Estudos subsequentes devem ser conduzidos com desenho que contemple cálculo amostral para melhor exploração dessas associações, permitindo maiores generalizaçôes. Embora a literatura traga que medicamentos opioides e anticolinérgicos - amplamente utilizados no ato anestésico cirúrgico - possam favorecer presença de sede, pois, causam ressecamento da cavidade oral, ${ }^{(13)}$ neste estudo essa associação não foi evidenciada. Estudos futuros devem contemplar a análise da influência das doses medicamentosas sobre a sede da criança. 
Em relação às contribuiçóes para a área de enfermagem, este estudo evidenciou o cenário de um desconforto prevalente e intenso que a criança cirúrgica vivencia, mas que tem sido negligenciado tanto em pesquisas como na prática clínica. A sede é um sintoma complexo, percebido e decodificado pela criança como um estressor, repercutindo em suas reações e recuperação pós-cirúrgica. É essencial compreender que a sede da criança cirúrgica é multifatorial e individual. Sua percepçáo e manejo pela equipe de enfermagem têm o potencial de afetar as repercussóes desse sintoma em uma fase crítica para a criança, como é a recuperação anestésica.

Deve-se encarar como prioridade e desafio elevar o estado de percepção da equipe que cuida da criança no que se refere à sede perioperatória. É primordial que protocolos e estratégias para manejá-la, já disponíveis em literatura, sejam incorporados no cuidado para com a criança cirúrgica.

\section{Conclusão}

A prevalência da sede em SRA foi muito elevada, levando-se em consideração que quase a metade das crianças referiu estar com sede desde o pré-operatório. A intensidade verbalizada foi predominante forte e intensa, demonstrando a magnitude deste sintoma para a criança. Quando em recuperação anestésica, é capaz de identificar os atributos da sede, particularmente boca seca e saliva grossa. Os fatores associados à maior intensidade da sede foram: sexo feminino, queixa espontânea da sede, referir sensação de boca seca e de saliva grossa, e menor idade. Não foi encontrada associação estatisticamente significativa entre variáveis preditoras, como tempo de jejum e uso de medicaçóes com presença e intensidade da sede.

\section{Colaborações}

Riviera A, Pierotti I, Mello CRL, Birolim MM e Fonseca LF contribuíram com a concepção do projeto, análise e interpretação dos dados, redação do artigo, revisão crítica relevante do conteúdo intelectual e aprovação final da versão a ser publicada.

\section{Referências}

1. Carvalho R, Bianchi ER. Enfermagem em Centro Cirúrgico e Recuperação. 2a ed. Barueri: Manole; 2016.

2. Frykholm P, Schindler E, Sümpelmann R, Walker R, Weiss $M$. Preoperative fasting in children: Review of existing guidelines and recent development. Br J Anaesth. 2018;120(3):469-74. Review.

3. American Society of Anesthesiologists (ASA). Practice guidelines for preoperative fasting and the use of pharmacologic agents to reduce the risk of pulmonary aspiration: application to healthy patients undergoing elective procedures. Anesthesiolog. 2017;126(3):376-93.

4. Chauvin C, Schalber-Geyer AS, Lefebvre F, Bopp C, Carrenard G, Marcoux L, et al. Early postoperative oral fluid intake in paediatric day case surgery influences the need for opioids and postoperative vomiting: a controlled randomized trial. Br J Anaesth. 2017;118(3):407-414.

5. Dolgun E, Yavuz M, Eroğlu B, Islamoğlu A. Investigation of preoperative fasting times in children. J Perianesth Nurs. 2017;32(2):121-4.

6. Engelhardt T, Wilson G, Horne L, Weiss M, Schmitz A. Are you hungry? Are you thirsty?-fasting times in elective outpatient pediatric patients. Paediatr Anaesth. 2011;21(9):964-8.

7. Campana MC, Fonseca LF, Lopes DFM, Martins PR. Percepção dos cuidadores quanto à sede da criança cirúrgica. Rev Rene. 2015;16(6):799-808.

8. Moore GH. Improving hydration in children: a sensible guide. Nutr Bull. 2013;38(2):236-42.

9. Al-Robeye AM, Barnard AN, Bew S. Thirsty work: Exploring children's experiences of preoperative fasting. Paediatr Anaesth. 2020;30(1):439.

10. Brady M, Kinn S, Ness V, O'Rourke K, Randhawa N, Stuart P. Preoperative fasting for preventing perioperative complications in children. Cochrane Database Syst Rev. 2009;(4):CD005285.

11. Thornton SN. Thirst and hydration: physiology and consequences of dysfunction. Physiol Behav. 2010;100(1):15-21.

12. Arai S, Stotts N, Puntillo K. Thirst in critically ill patients: from physiology to sensation. Am J Crit Care. 2013;22(4):328-35. Review.

13. Ortenzi AV. Medicação pré-anestésica. In: Cangiani LM, Carmona MJ, Torres ML, Bastos CO, Ferez D, Silva ED, et al. Tratado de anestesiologia. $6^{a}$ ed. São Paulo: Atheneu; 2006. v. 2. p.1044.

14. Steward DJ. A simplified scoring system for the post-operative recovery room. Can J Anaesth. 1975;22(1):111-3.

15. Piaget J. Seis estudos de psicologia. 24a ed. Rio de Janeiro: Forense Universitária; 1999.

16. Claro MT, Vietta EP. Escala de faces para avaliação da dor em crianças - etapa preliminar [tese]. Ribeirão Preto: Universidade de São Paulo, Escola de Enfermagem de Ribeirão Preto; 1993.

17. Martins PR, Fonseca LF. Avaliação das dimensões da sede: revisão integrativa. Rev Eletr Enferm. 2017;19:a09.

18. Nascimento LA, Nakaya TG, Conchon MF, Garcia AK, Pierotti I, Serato VM, et al. Prevalência, intensidade e desconforto da sede no paciente cirúrgico no pós-operatório imediato. Rev SOBECC. 2019;24(2):85-90. 
19. Edmonds CJ, Burford D. Should children drink more water? The effects of drinking water on cognition in children. Appetite. 2009;52(3):776-79.

20. Thomas M, Morrison C, Newton R, Schindler E. Consensus statement on clear fluids fasting for elective pediatric general anesthesia. Paediatr Anaesth. 2018;28(5):411-4.

21. Pierotti I, Fracarolli IFL, Fonseca LF, Aroni P. Avaliação da intensidade e desconforto da sede perioperatória. Esc Anna Nery. 2018;22(3):e20170375.

22. Dood M, Janson S, Facione N, Faucett J, Froelicher ES, Humphreys $\mathrm{J}$, et al. Advancing the science of symptom management. J Adv Nurs. 2001;33(5):668-76.

23. Conchon MF, Nascimento LA, Fonseca LF, Aroni P. Perioperative thirst: an analysis from the perspective of the Symptom Management Theory. Rev Esc Enferm USP. 2015;49(1):122-28.

24. Benelam B. Recognizing the signs of dehydration. Pract Nurs. 2010;21(5):230-36.

25. Brunet-Wood K, Simons M, Evasiuk A, Mazurak V, Dicken B, Ridley D, et al. Surgical fasting guidelines in children: Are we putting them into practice? J Pediatr Surg. 2016;51:1298-302.
26. Schmtz A, Kellenberger CJ, Neuhaus D, Schoroeter E, Deanovic D, Prüfer $F$, et al. Fasting times and gastric contents volume in children undergoing deep propofol sedation - an assessment using magnetic resonance imaging. Paediatr Anaesth. 2011;21(6):685-90.

27. Klemetti S, Kinnunen I, Suominen T, Antila H, Vahlberg T, Grenman R, et al. The effect of preoperative fasting on postoperative thirst, hunger and oral intake in paediatric ambulatory tonsillectomy. J Clin Nurs. 2010;19(3-4):341-50

28. Stevenson RJ, Mahmut M, Rooney K. Individual differences in the interoceptive states of hunger, fullness and thirst. Appetite. 2015;95:44-57.

29. Martins PR, Fonseca LF, Rossetto EG, Mai LD. Elaboração e validação de Escala de Desconforto da Sede Perioperatória. Rev Esc Enferm USP. 2017;51:e03240.

30. Silva LC, Aroni P, Fonseca LF. Tenho sede! Vivência do paciente cirúrgico no período perioperatório. Rev SOBECC. 2016;21(2):7581.

31. D'Anci KE, Constant F, Rosenberg $\mathrm{H}$. Hydration and cognitive function in children. Nutr Rev. 2006;64(10 Pt 1):457-64. Review. 